\title{
PERFIL EPIDEMIOLÓGICO DE CASOS DE HANSENÍASE EM UM ESTADO DO NORDESTE BRASILEIRO
}

\author{
EPIDEMIOLOGICAL PROFILE OF LEPROSY CASES IN A NORTHEAST BRAZILIAN STATE
}

\author{
Mísia Joyner de Sousa Dias Monteiro , Gleyson Moura dos Santos ${ }^{\mathrm{b}}$, \\ Maryanna Tallyta Silva Barretoc, Renata Vieira de Sousa Silva ${ }^{d}$, Ruan Luiz Rodrigues de Jesuse, \\ Higo José Neri da Silva ${ }^{f}$

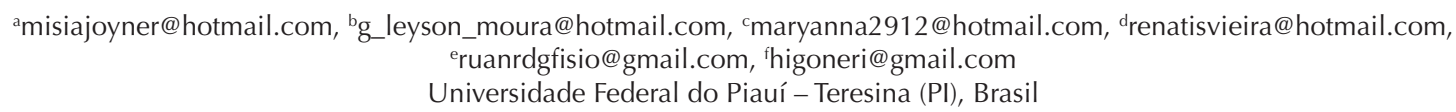

\section{RESUMO}

Introdução: A hanseníase é uma doença infecciosa crônica, causada pelo Mycobacterium leprae, sendo considerada um grande problema de saúde pública nos países em desenvolvimento. Objetivo: Apresentar as características epidemiológicas, clínicas e a distribuição espacial de casos de hanseníase no estado do Piauí entre os anos de 2011 a 2015. Materiais e métodos: Trata-se de um estudo descritivo de base populacional, utilizando dados secundários de hanseníase registrados no Sistema de Informações de Agravos de Notificação e disponibilizados no site do Departamento de Informático do Sistema Único de Saúde. Resultados e conclusóes: Foram notificados 6.378 casos, com incidência média de 0,63 casos/100.000 habitantes. Os casos por faixa etária variaram entre 35 e 49 anos para o sexo feminino e entre 50 e 64 anos para o sexo masculino. O modo de detecção foi feito em 43,5\% dos casos através de encaminhamento. 69,3\% dos pacientes obtiveram cura. A classificação operacional multibacilar prevaleceu para o sexo masculino e paucibacilar para o sexo feminino. $67,1 \%$ dos pacientes não apresentavam nenhum grau de incapacidade física e apresentavam de 2 a 5 lesões. 38,5\% fazia uso de esquema terapêutico com $\mathrm{PQT} / \mathrm{PB} / 6$ doses e $60,3 \%$ faziam com PQT/MB/12doses. A maior ocorrência dos casos no ano de 2011 foi observada no município Pedro Laurentino; em 2012 no município de Passagem Franca do Piauí; 2013 no município de Flores do Piauí; 2014 no município de Cristino Castro e em 2015 no município de Santa Rosa do Piauí. Conclui-se que a hanseníase é uma doença grave, muitas vezes negligenciada, e que requer atenção especial por parte das autoridades de saúde pública.

Palavras-chave: Doenças transmissíveis; epidemiologia; saúde pública.

\section{ABSTRACT}

Introduction: Leprosy is a chronic infectious disease caused by Mycobacterium leprae. It is considered a major public health issue in developing countries. Objective: To present the epidemiological and clinical characteristics and the spatial distribution of leprosy cases in the state of Piauí between the years of 2011 and 2015. Materials and methods: This is a descriptive and population-based study, using secondary data of leprosy cases registered in the Information System on Diseases of Compulsory Declaration (Sinan) and made available on the Datasus website. Results and conclusions: There were 6378 cases reported, with an average incidence of 0.63 cases per 100,000 inhabitants. The cases by age group ranged among 35 to 49 years for females and 50 to 64 for males. The detection mode was performed in $43.5 \%$ of the cases by referral and $69.3 \%$ of patients were cured. The multibacillary operational classification prevailed for males and the paucibacillary for females. $67.1 \%$ of the patients did not present any degree of physical disability and had 2 to 5 lesions. $38.5 \%$ were in a therapeutic regimen with MDT/ $\mathrm{PB} / 6$ doses and $60.3 \%$ had MDT/MB/12doses. The highest occurrence of cases in 2011 was observed 
in the city of Pedro Laurentino, in 2012 in Passagem Franca do Piauí, 2013 in Flores do Piauí, 2014 in Cristino Castro and 2015 in Santa Rosa do Piauí. In conclusion, leprosy is a serious disease often neglected that requires particular attention from public health authorities.

Keywords: Communicable diseases; epidemiology; public health.

\section{Introdução}

A hanseníase é uma enfermidade infectocontagiosa de evolução crônica que acomete o homem, sendo de difícil diagnóstico e tempo de incubação prolongado, variando de 2 a 10 anos; alta contagiosidade e baixa morbidade. Essa patologia é causada pelo parasita intracelular obrigatório Mycobacterium leprae (bacilo de Hansen), e o contágio ocorre através de uma pessoa infectada pelo bacilo na forma multibacilar não tratada, que o elimina pelo meio exterior através das vias respiratórias superiores, contagiando pessoas susceptíveis à doença ${ }^{1,2,3}$.

As diferentes formas clínicas da hanseníase estão relacionadas com a imunogenicidade do bacilo e com o sistema imunológico do hospedeiro, com sinais e sintomas dermatoneurológicos, lesóes na pele e nervos periféricos, principalmente nos olhos, mãos e pés, com potencial para provocar deformidades quando não tratada ou tratada tardiamente. Consequentemente, observa-se que isso contribui para regressão da capacidade do trabalho, limitação da vida social e problemas psicológicos, sendo também responsáveis pelo estigma e preconceito da doença ${ }^{4,5}$.

O Ministério da Saúde divulga números que apontam redução de $34,1 \%$ no número de casos novos diagnosticados com hanseníase no Brasil, passando de 43.652 diagnosticados no ano de 2006, para 28.761 no ano de 2015. Tal reduçáo corresponde assim à queda de $39,7 \%$ da taxa de detecção geral do país, que passou de 23,37/100 mil habitantes em 2006 para 14,07/100 mil habitantes em 2015. A redução é resultado das açoóes implantadas no país para o enfrentamento da doença, com foco na busca ativa de casos novos para o diagnóstico na fase inicial, tratamento oportuno e cura, bem como a prevenção de incapacidades e deformidades físicas, principal causa do estigma e preconceito que permeiam a doença. Em menores de 15 anos, o número de casos novos da doença diagnosticados em 2015 foi de 2.113, sinalizando assim focos de infecção ativos e transmissão recente. Contudo, a taxa de detecção geral nessa parcela da população apresentou uma redução acumulada de $28,2 \%$ na última década, passando de 6,22/100 mil habitantes em 2006 para 4,46/100 mil habitantes em $2015^{6}$.

O diagnóstico da hanseníase é determinado pela classificação operacional dos casos. Esta toma por base o número das lesốes na pele, até cinco ou mais de cinco lesōes, classificando, respectivamente, o caso em formas paucibacilar (PB) e multibacilar (MB). Quando realizada a baciloscopia com resultado positivo, o caso é classificado como MB independentemente do número de lesôes. A negatividade do exame não exclui o diagnóstico de hanseníase nem classifica o caso como PB. A classificaçáo operacional direciona o esquema do tratamento poliquimioterápico a ser estabelecido para o paciente ${ }^{7}$.

Frente a esse cenário, a Organização Pan-Americana da Saúde - OPAS caracteriza a hanseníase como uma doença negligenciada e relacionada à pobreza, que afeta proporcionalmente mais indivíduos na faixa economicamente ativa, levando à instalação de incapacidades, ao descumprimento das metas estabelecidas para redução da endemia, à perda de força laboral e à onerosidade da renda pública ${ }^{8}$. Desta forma, diante do abordado, este estudo visou apresentar características epidemiológicas, diagnósticos clínicos e distribuição espacial de casos de hanseníase no estado do Piauí entre os anos de 2011 e 2015.

\section{Metodologia}

Trata-se de um estudo descritivo/retrospectivo de base populacional, utilizando dados secundários de casos de hanseníase ocorridos entre os anos de 2011 e 2015 no estado do Piauí, registrados no Sistema de Informaçôes de Agravos de Notificação (SINAN) e disponibilizados no site do Departamento de Informática do Sistema Único de Saúde (DATASUS).

As variáveis incluídas para análise neste estudo foram: sexo, faixa etária, modo de detecção, classificação operacional, avaliação da incapacidade física no momento do diagnóstico, quantidades de lesóes cutâneas, esquema terapêutico e tipo de alta.

Calculou-se o número de casos autóctones ocorridos por município entre os anos de 2011 e 2015 . Realizouse a produção do mapa de distribuição dos casos de hanseníase por município do estado, dividindo os valores obtidos em quintis para a classificação dos municípios.

Para tabulação, análise dos dados e produção dos mapas foi utilizado os programas Tabwin 3.6 e o Microsoft Office Excel 2010. As estimativas populacionais empregadas para o cálculo do coeficiente de incidência 
por 100.000 habitantes foram obtidas do Instituto Brasileiro de Geografia e Estatística (IBGE). O software Tabwin 3.6 e a base cartográfica digital para a produção do mapa foram obtidos no site do DATASUS.

Os dados foram analisados mediante estatísticas descritivas (frequências absolutas, gráficos e tabelas). Para as comparações das diferenças nas frequências das variáveis entre os sexos, utilizou-se o teste do qui-quadrado com correção de Yates para tabelas $2 \times 2$. O tratamento dos dados foi feito através do programa Bioestat 5.0, sendo considerado significativo $\mathrm{p}<0,05$.

Como o estudo trata-se de uma análise fundamentada em banco de dados secundários de domínio público, este não foi encaminhado para apreciação de um Comitê de Ética em Pesquisa, no entanto ressalta-se que foram tomados os cuidados éticos que preceituam a Resolução 466/12, do Conselho Nacional de Saúde?.

\section{Resultados}

No período de 2011 a 2015, foram notificados 6.378 casos de hanseníase no estado do Piauí, com média de 1.275,6 casos anualmente. As maiores frequências observadas foram nos anos de 2011 ( $\mathrm{n}=1358 \mathrm{ca}$ sos, $21,3 \%)$ e 2014 ( $\mathrm{n}=1308$ caos, $20,5 \%)$, e a menor ocorrência foi registrada no ano de 2015 (n=1194 casos, 18,7\%) (Figura1).

Figura 1: Distribuição do número de casos notificados de hanseníase no estado do Piauí entre os anos de 2011 a 2015.

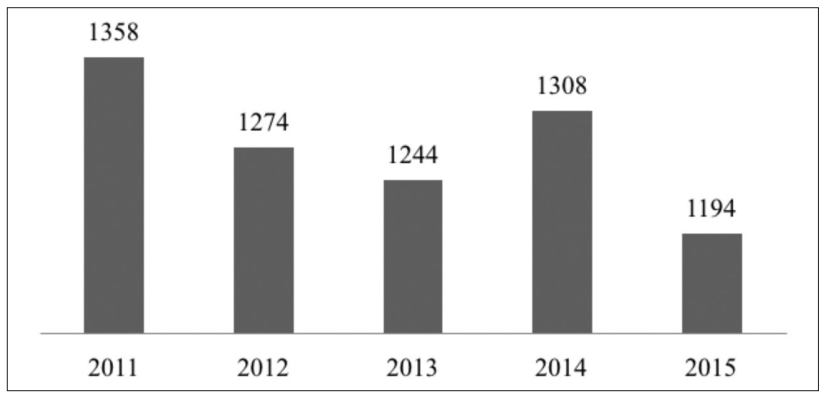

Fonte: Ministério da Saúde/SVS - Sistema de Informação de Agravos de Notificaçấo - SINAN NET, 2017.

Referente ao coeficiente de incidência, a média no período foi de 0,63 casos/ 100.000 habitantes. Durante os 5 anos analisados, foi contabilizada a incidência de casos anualmente: 0,69 casos/100.000 habitantes no ano de 2011; 0,64 casos/100.000 habitantes no ano de 2012; 0,62 casos/100.000 habitantes no ano de 2013; 0,65 casos/100.000 habitantes no ano de 2014; 0,58 casos/100.000 habitantes no ano de 2015 (Figura 2).
Figura 2: Distribuição do coeficiente de incidência dos casos de hanseníase/100.000 habitantes no estado do Piauí entre os anos de 2011 a 2015.

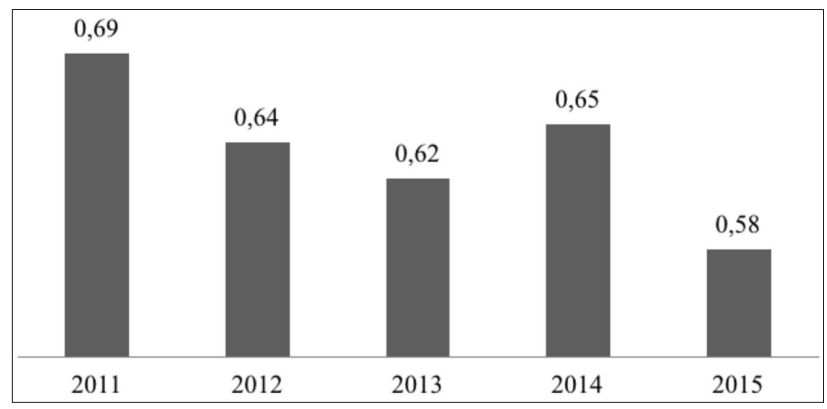

Fonte: Ministério da Saúde/SVS - Sistema de Informação de Agravos de Notificação - SINAN NET, 2017.

Com relação à ocorrência de casos por sexo, a Tabela 1 mostra que 3.488 casos $(54,7 \%)$ eram de indivíduos do sexo masculino, sendo este grupo mais prevalente quando comparado ao do sexo feminino.

Tabela 1: Distribuição por sexo de casos de hanseníase confirmados em residentes no estado do Piauí entre os anos de 2011 a 2015 .

\begin{tabular}{lcc} 
Sexo & Número de casos $(\mathbf{n}=\mathbf{6 3 7 8})$ & $\%$ \\
Masculino & 3488 & 54,7 \\
Feminino & 2890 & 45,3 \\
\hline
\end{tabular}

Fonte: Ministério da Saúde/SVS - Sistema de Informação de Agravos de Notificação - SINAN NET, 2017.

No tocante à distribuição da hanseníase por faixa etária, avaliou-se as seguintes classificaçóes: < 15 anos, 15 a 19, 20 a 34, 35 a 49, 50 a 64 e > 65 anos (Figura 3). A faixa etária predominante variou entre os sexos, registrando-se maior número de casos femininos entre 35 e 49 anos, com 763 casos $(26,4 \%)$ e maior número de casos masculinos na faixa etária de 50 a 64 anos, com 879 casos $(25,2 \%)$.

Figura 3: Distribuição por faixas etárias de pacientes com hanseníase segundo o sexo, no estado do Piauí entre os anos de 2011 a 2015.

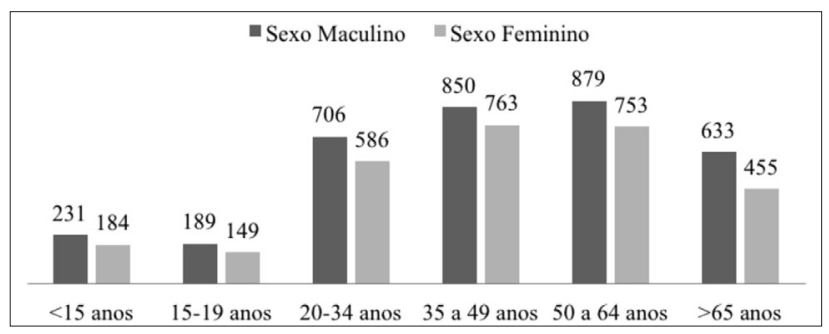

Fonte: Ministério da Saúde/SVS - Sistema de Informação de Agravos de Notificação - SINAN NET, 2017. 
A detecção, entre os anos de 2011 e 2015, foi feita em $43,5 \%$ dos casos, pela forma de encaminhamento, com diferença estatística entre os sexos $(\mathrm{p}<0,0268)$, e $33,9 \%$ por demanda espontânea, sem diferença estatística entre os sexos $(\mathrm{p}=0.4014)$. A porcentagem de pacientes que obtiveram cura foi de $69,3 \%$, com diferença estatística entre os sexos $(\mathrm{p}<0,0001)$.

A distribuição dos casos, segundo a classificação operacional e o sexo, foi classificada como multibacilares para o sexo masculino $(\mathrm{p}<0,0001)$ e paucibacilares para o sexo feminino $(\mathrm{p}<0,0001)$. A avaliação das incapacidades físicas revelou que 2.168 pacientes do sexo masculino $(62,1 \%)$ e 2.109 do sexo feminino $(73 \%)$ não apresentavam nenhum grau de incapacidade no momento do diagnóstico $(\mathrm{p}<0,0001)$. Quanto ao número de lesóes cutâneas, a maioria dos casos $(\mathrm{n}=2.022$, $31,7 \%$ ) apresentava de 2 a 5 lesóes, sem diferença estatística entre os sexos $(\mathrm{p}=0.5064)$.

Dentre os 6.378 casos registrados nos anos estudados, $2.453(38,5 \%)$ faziam uso de esquema terapêutico com PQT/PB/6doses e 3.848 (60,3\%) faziam uso de esquema terapêutico com PQT/MB/12doses, apresentando diferença estatística entre os sexos $(\mathrm{p}<0,0001)$. As principais características clínicas e epidemiológicas do grupo identificado separados por sexo estão na Tabela 2.

Tabela 2: Características clínicas e epidemiológicas de pacientes com hanseníase segundo o sexo, no estado do Piauí entre os anos de 2011 a 2015.

\begin{tabular}{|c|c|c|c|c|c|c|}
\hline \multirow{2}{*}{ Características } & \multicolumn{2}{|c|}{ Homens $(n=3488)$} & \multicolumn{2}{|c|}{ Mulheres $(\mathrm{n}=\mathbf{2 8 9 0})$} & \multirow{2}{*}{$X^{2}$} & \multirow{2}{*}{ Valor de $\mathbf{p}$} \\
\hline & $(\mathbf{n})$ & $(\%)$ & $(\mathbf{n})$ & $(\%)$ & & \\
\hline \multicolumn{7}{|l|}{ Classificação Operacional } \\
\hline Multibacilar & 2468 & 70,8 & 1384 & 47,9 & 344,6 & $<0,0001^{*}$ \\
\hline Paucibacilar & 1020 & 29,2 & 1506 & 52,1 & 344,6 & $<0,0001^{*}$ \\
\hline \multicolumn{7}{|l|}{ Avaliação da Incapacidade } \\
\hline Grau 0 & 2168 & 62,1 & 2109 & 73,0 & 83,3 & $<0,0001^{*}$ \\
\hline Grau 1 & 763 & 21,9 & 495 & 17,1 & 22,2 & $<0,0001^{*}$ \\
\hline Grau 2 & 271 & 7,8 & 94 & 3,3 & 58,9 & $<0,0001^{*}$ \\
\hline Sem Informação & 286 & 8,2 & 192 & 6,6 & 5,3 & $0,0214^{*}$ \\
\hline \multicolumn{7}{|l|}{ Número de Lesóes } \\
\hline Nenhuma Lesão & 99 & 2,9 & 66 & 2,3 & 1,7 & 0,1903 \\
\hline Lesão Única & 707 & 20,3 & 1011 & 35,0 & 173,1 & $<0,0001^{*}$ \\
\hline 2 a 5 Lesóes & 1093 & 31,3 & 929 & 32,1 & 0,4 & 0,5064 \\
\hline$>5$ Lesóes & 741 & 21,2 & 413 & 14,3 & 51,1 & $<0,0001^{*}$ \\
\hline Sem Informação & 848 & 24,3 & 471 & 16,3 & 61,4 & $<0,0001^{*}$ \\
\hline \multicolumn{7}{|l|}{ Esquema Terapêutico } \\
\hline PQT/PB/6 doses & 996 & 28,6 & 1457 & 50,4 & 318,2 & $<0,0001^{*}$ \\
\hline PQT/MB/12 doses & 2459 & 70,5 & 1389 & 48,1 & 331,5 & $<0,0001^{*}$ \\
\hline Outros esquemas alternativos & 22 & 0,6 & 28 & 1,0 & 1,9 & 0,1671 \\
\hline Sem Informação & 11 & 0,3 & 16 & 0,5 & 1,6 & 0,2058 \\
\hline \multicolumn{7}{|l|}{ Modo de Deteç̧áo } \\
\hline Encaminhamento & 1474 & 42,3 & 1302 & 45,1 & 4,9 & $0,0268^{*}$ \\
\hline Demanda Espontânea & 1197 & 34,3 & 962 & 33,3 & 0,7 & 0,4014 \\
\hline Exame Coletividade & 99 & 2,8 & 117 & 4,0 & 6,7 & $0,0096^{*}$ \\
\hline Exame Contato & 91 & 2,6 & 99 & 3,4 & 3,4 & 0,0664 \\
\hline Outros Modos & 73 & 2,1 & 49 & 1,7 & 1,1 & 0,2884 \\
\hline Sem Informação & 554 & 15,9 & 361 & 12,5 & 14,5 & $0,0001^{*}$ \\
\hline \multicolumn{7}{|l|}{ Tipo de Alta } \\
\hline Cura & 2343 & 67,2 & 2080 & 72,0 & 16,9 & $<0,0001^{*}$ \\
\hline Transferido para o mesmo Município & 22 & 0,6 & 17 & 0,6 & 0,003 & 0,9558 \\
\hline Transferido para outro Município & 191 & 5,5 & 115 & 4,0 & 7,4 & $0,0064^{*}$ \\
\hline Transferido para outro Estado & 50 & 1,4 & 38 & 1,3 & 0,088 & 0,7669 \\
\hline Óbito & 64 & 1,8 & 21 & 0,7 & 13,9 & $0,0002^{*}$ \\
\hline Abandono & 104 & 3,0 & 69 & 2,4 & 1,9 & 0,1687 \\
\hline Erro Diagnóstico & 40 & 1,1 & 47 & 1,6 & 2,3 & 0,1748 \\
\hline Sem Informação & 674 & 19,4 & 503 & 17,4 & 3,7 & 0,0531 \\
\hline
\end{tabular}

*Valor de p significativo <0,05. PQT- Poliquimioterapia; PB- Esquema Paucibacilar; MB- Esquema Multibacilar.

Fonte: Ministério da Saúde/SVS - Sistema de Informação de Agravos de Notificação - SINAN NET, 2017. 
No que concerne à distribuição espacial dos casos de hanseníase por município no estado do Piauí, a maior ocorrência de casos no ano de 2011 foi observada no município Pedro Laurentino com 204,92 casos/100.000 habitantes; em 2012 no município de Passagem Franca do Piauí com 202,61 casos/100.000 habitantes
(Figura 4). No ano de 2013 no município de Flores do Piauí com 181,98 casos/100.000 habitantes; em 2014 no município de Cristino Castro com 343, 54 casos/100.000 habitantes (Figura 5). E em 2015 no município de Santa Rosa do Piauí apresentando 270,37 casos/100.000 habitantes (Figura 6).

Figura 4: Distribuição espacial dos casos de Hanseníase por município do estado do Piauí nos anos de 2011 e 2012.

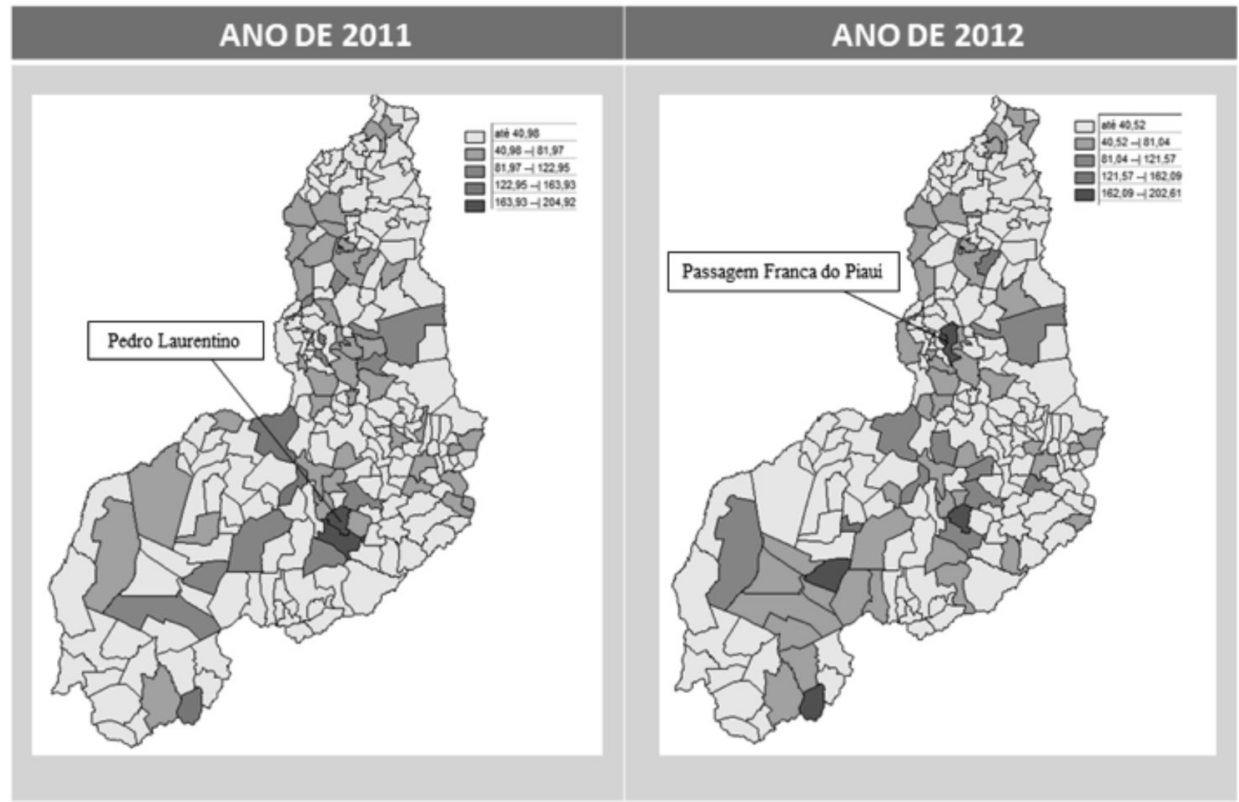

Fonte: Ministério da Saúde/SVS - Sistema de Informação de Agravos de Notificação - SINAN NET, 2017.

Figura 5: Distribuição espacial dos casos de Hanseníase por município do estado do Piauí nos anos de 2013 e 2014.

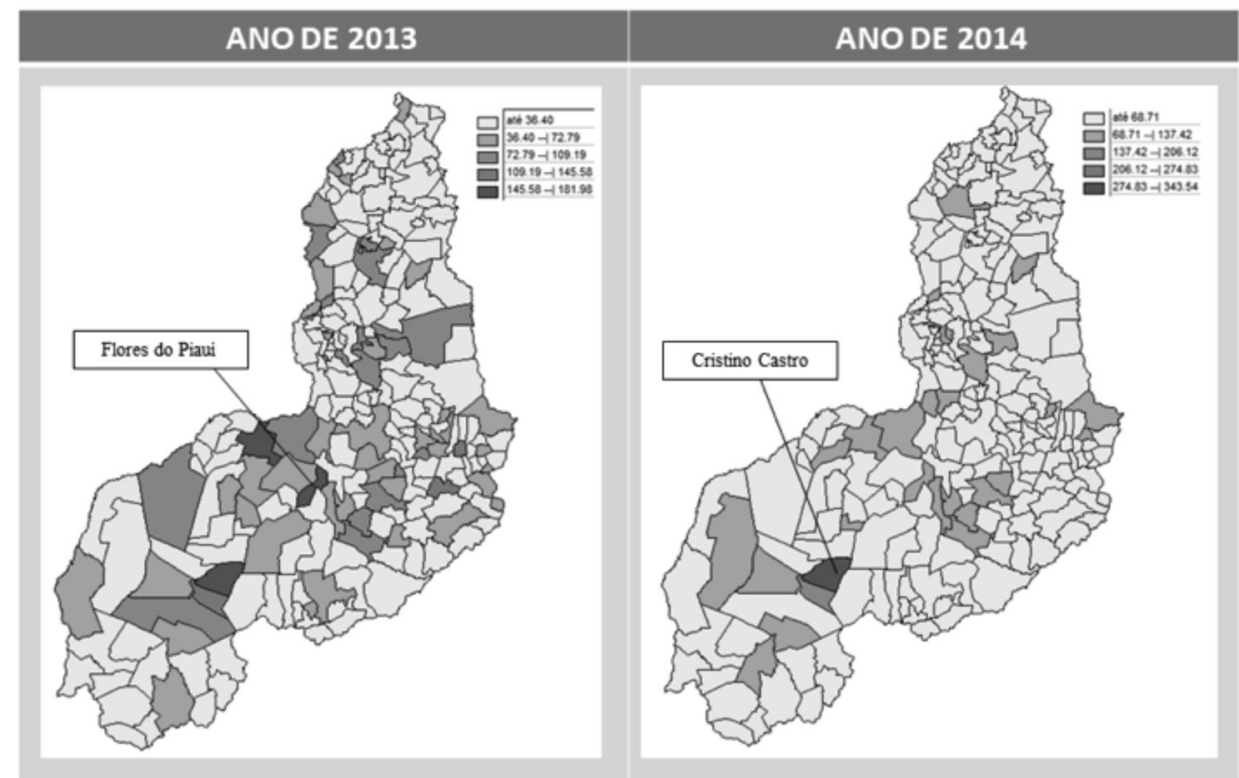

Fonte: Ministério da Saúde/SVS - Sistema de Informação de Agravos de Notificação - SINAN NET, 2017. 
Figura 6: Distribuição espacial dos casos de Hanseníase por município do estado do Piauí no ano de 2015.

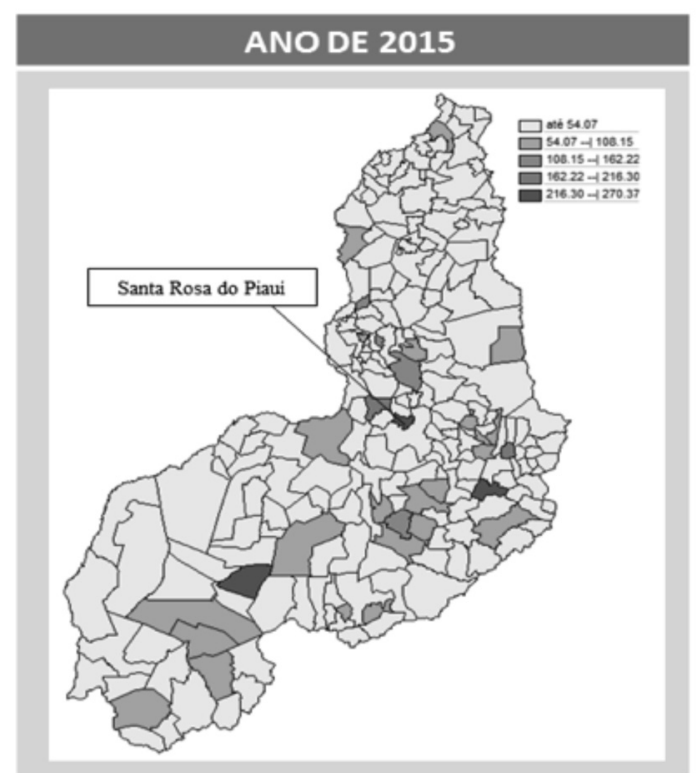

Fonte: Ministério da Saúde/SVS - Sistema de Informaçấo de Agravos de Notificação - SINAN NET, 2017.

\section{Discussão}

Este estudo permitiu identificar o comportamento da hanseníase relacionado aos seus aspectos epidemiológicos, no estado do Piauí. Com relação aos números de casos observados no período temporal estudado, pode-se perceber que houve uma variação entre um ano e outro, resultado este semelhante ao obtido por Barbosa et al. ${ }^{10}$, que, ao estudar o perfil epidemiológico da hanseníase em uma cidade hiperendêmica no Maranhão, reportaram um percentual de casos notificados que variava de 6,72 a $19,57 \%$.

A meta de eliminação da hanseníase proposta pela Organização Mundial da Saúde (OMS) é de menos de 1 caso por 10 mil habitantes ${ }^{11}$. Analisando-se isoladamente cada ano de estudo, observou- se que, no período de 2011 a 2015, a incidência da hanseníase permaneceu entre 0,58 a 0,69 casos por 100 mil habitantes. Tais taxas estiveram bem abaixo do sugerido pela OMS, o que comprova o fortalecimento e a expansão das medidas de controle da doença.

Com relação aos casos de hanseníase entre os sexos durante o período analisado, o gênero masculino prevaleceu sobre o feminino, tal resultado é condizente com a literatura, em que esta apresenta vários estudos divergentes em relação à prevalência de hanseníase quanto ao sexo $^{12,13}$. Alguns autores comprovam que o maior contato social entre homens e sua frequente exposição a ambientes de risco favorece o aumento do número de casos, enquanto que a menor preocupação com a estética corporal e a falta de políticas específicas para esse grupo pode contribuir na deficiência do diagnóstico, o que justificaria o predomínio do sexo feminino em alguns estudos ${ }^{14,15}$.

De acordo com os dados levantados quanto a faixa etária, o estudo revela que a hanseníase atingiu predominantemente os adultos, resultado semelhante foi demonstrado por Barbosa et al. ${ }^{10}$, no qual os autores em seu estudo apresentaram concentração de idades entre 20 e 39 anos $(33,95 \%)$, esse intervalo relaciona-se com o período economicamente produtivo e caracteriza a hanseníase como doença de adultos e adultos jovens. Segundo Norman et al. ${ }^{16}$, a hanseníase é considerada uma doença de adultos pelo longo período de incubação, entretanto as crianças também são suscetíveis. Assim, quando ocorrem casos na família em áreas endêmicas, o risco de crianças adoecerem aumenta. A ocorrência de hanseníase em crianças é considerada um indicador da prevalência da doença na população geral, e sua detecção reflete circuitos de transmissão ativos.

Quanto à classificação operacional, infere-se que a ocorrência de casos multibacilares no estado do Piauí foi preeminente durante o período analisado. Este fato foi também demonstrado em um estudo realizado na cidade de Montes Claros (MG) no período de 2009 a 2013 e outro na cidade do Nordeste brasileiro nos anos de 2010 e $2011^{17,18}$.

Ribeiro Júnior, Vieira e Caldeira ${ }^{19}$ afirmam em seu estudo que o portador multibacilar está associado a uma chance nove vezes maior em desenvolver algum grau de incapacidade física. Dessa forma, tais levantamentos acabam gerando preocupação em relação a esses resultados, uma vez que os pacientes multibacilares são a principal fonte de infecção da doença e os mais suscetíveis à enfermidade.

A hanseníase, além de ser um problema de saúde pública devido ao grande número de casos, tem um alto potencial de causar incapacidades físicas, interferindo na fase produtiva e na vida social do paciente, determinando perdas econômicas e traumas psicológicos. Essas incapacidades têm sido ponderadas pelo estigma e pela discriminação dos pacientes, portanto Pereira et al. ${ }^{20}$ relatam que uma das formas mais eficazes de determinar se o diagnóstico tem sido precoce é identificando a presença de incapacidades físicas no momento da diagnose. $\mathrm{O}$ grau de incapacidade é determinado a partir da avaliação neurológica dos olhos, das mãos e dos pés, e o seu resultado é expresso em valores que variam de zero a dois, sendo zero se não houver comprometimento neural; um para 
diminuição ou perda da sensibilidade e dois para presença de incapacidades e deformidades ${ }^{21}$. Em relaçáo à avaliação da incapacidade, os dados deste estudo demonstraram que a maioria dos pacientes apresentou, por ocasiáo do diagnóstico, grau zero de incapacidade física, fato este que entra em consonância com uma pesquisa realizada no município de Montes Claros. Nesta, quando analisado o grau de incapacidade, $77,41 \%$ dos pacientes não apresentou incapacidade física (grau zero) ${ }^{17}$. Averiguando esses achados, pode-se inferir que o diagnóstico tem se tornado prévio e as estratégias de prevenção e controle da patologia têm sido eficaz no âmbito da saúde pública, assim, contribuindo para a regressão de morbimortalidade.

Quanto ao número de lesóes cutâneas apresentadas pelos pacientes no momento do diagnóstico, observou- se que a maioria dos indivíduos apresentaram de duas a cinco lesões cutâneas. Os mesmos resultados foram apresentados no estudo de Ribeiro Júnior, Vieira e Caldeira ${ }^{19}$, no qual todos os pacientes estudados apresentaram o mesmo número de lesóes cutâneas, reportados por este estudo.

Observou-se que 43 (5,3\%) dos diagnósticos foram feitos através da demanda espontânea, ou seja, foi a população que procurou o serviço de saúde, fato também observado por Miranzi, Pereira e Nunes ${ }^{13}$. Em relação ao esquema terapêutico, foi possível notar que $98,8 \%$ das pessoas vinham fazendo uso de poliquimioterapia, o que acaba refletindo nos resultados de cura, fator observado também por este estudo. Tal situação caracteriza positivamente o processo de integração das açóes de controle da hanseníase nos serviços de atenção primária, de modo a produzir melhores resultados e solidificar o controle da doença.

O padrão de ocorrência nos municípios do estado do Piauí ilustra claramente o processo de interiorização da hanseníase. O estudo da distribuição por áreas destaca a distribuição geográfica da doença, o que possibilita a visualização de áreas de aglomeração de focos da doença, indicando as áreas cuja necessidade de atenção nas atividades de vigilância epidemiológica é maior. Para Barcellos e Bastos ${ }^{22}$, estes elementos visuais facilitam a interpretação, com a possibilidade de indicadores de risco para a doença.

\section{Conclusão}

Com a realização deste estudo, foi possível identificar o comportamento da hanseníase relacionado aos seus aspectos clínicos e epidemiológicos no estado do Piauí. A hanseníase é uma doença grave e, muitas vezes, negligenciada, que requer atenção especial por parte das autoridades de saúde pública.
Embora no Brasil tenha-se realizado a detecção precoce, a busca ativa e o acompanhamento adequado, o que contribuiu para o avanço no enfrentamento da doença no país, há uma estabilidade do Estado para com a falta de comprometimento de colocar em prática as políticas públicas voltadas para educação em saúde para a população, a fim de colaborar com a redução da hanseníase no País e com a consequente regressão da endemicidade dessa patologia.

Portanto, faz- se necessária a contribuição do Estado em mobilizar os recursos que, de fato, possam ser investidos na sociedade, assim sendo eficaz o controle da doença, desde que também tenham profissionais capacitados nas instituiçóes de serviços realizando um trabalho digno e pleno para os usuários.

\section{Referências}

1. Lastória JC, Abreu MAMM. Hanseníase: diagnóstico e tratamento. Diagn Tratamento. 2012;17(4):173-9.

2. Rodrigues LC, Lockwood DN. Leprosy now: epidemiology, progress, challenges, and research gaps. Lancet Infect Dis. 2011;11(6):464-70.

3. Martins MVPS, Silva TS. Saúde pública e hanseníase na cidade de Uberlândia. OBSERVATORIUM: Rev Eletr Geogr. 2011;3(7):38-52.

4. Brasil. Ministério da Saúde. Gabinete do Ministro. Portaria no 3.125, de 7 de outubro de 2010. Aprova as diretrizes para vigilância, atenção e controle da hanseníase. Diário Oficial da União. Brasília, DF; 15 out 2010. Seção 1, p. 55.

5. Brasil. Ministério da Saúde. Secretária de Políticas de Saúde. Departamento de Atenção Básica. Guia para o controle de hanseníase. Brasília, DF: Ministério da Saúde; 2002.

6. Brasil. Ministério da Saúde. Portal da Saúde. Em 10 anos, o número de casos novos da doença caiu 34\%. [Internet]. Brasília, DF: Ministério da Saúde; 2017. [citado em 2017 maio 27]. Disponível em: https://goo.gl/1zS5i2.

7. Brasil. Ministério da Saúde. Secretaria de Vigilância em Saúde. Departamento de Vigilância das Doenças Transmissíveis. Diretrizes para vigilância, atençâo e eliminação da hanseníase como problema de saúde pública. Brasília, DF: Ministério da Saúde; 2016.

8. Pan American Health Organization; World Health Organization. 49th Directing Council: 61st Session of the Regional Committee. Washington, DC; 2009.

9. Brasil. Ministério da Saúde. Conselho Nacional de Saúde. Resolução no 466, de 12 de dezembro de 2012. Diretrizes e normas regulamentadoras de pesquisas envolvendo seres humanos. Diário Oficial da União. Brasília, DF; 13 jun 2013. Seção 1, p. 59-62. 
10. Barbosa DRM, Araújo AA, Damaceno JCF, Almeida MG, Santos AG. Perfil epidemiológico da hanseníase em cidade hiperendêmica do Maranhão, 2005-2012. Rev Acad Rede Cuid Saúde. 2014;8(1):1-13.

11. Brasil. Ministério da Saúde. Secretaria de Políticas de Saúde. Departamento de Atenção Básica. Hanseníase: atividades de controle e manual de procedimentos. Brasília, DF: Ministério da Saúde; 2001.

12. Lima HM, Sauaia N, Costa VRL, Coelho Neto GT, Figueiredo PMS. Perfil epidemiológico dos pacientes com hanseníase atendidos em Centro de Saúde em São Luís, MA. Rev Bras Clin Med. 2010;8(4):323-7.

13. Miranzi SSC, Pereira LHMP, Nunes AA. Perfil epidemiológico da hanseníase em um município brasileiro, no período de 2000 a 2006. Rev Soc Bras Med Trop. 2010;43(1):62-7.

14. Silva AR, Matos WB, Silva CCB, Gonçalves EGR. Hanseníase no município de Buriticupu, estado do Maranhão: busca ativa de casos na população adulta. Rev Soc Bras Med Trop. 2010;43(6):691-4.

15. Melão S, Blanco LFO, Mounzer N, Veronezi CCD, Simóes PWTA. Perfil epidemiológico dos pacientes com hanseníase no extremo sul de Santa Catarina, no período de 2001 a 2007. Rev Soc Bras Med Trop. 2011;44(1):79-84.
16. Norman G, Joseph GA, Udayasuriyan P, Samuel P, Venugopal M. Leprosy case detec $\neg$ tion using schoolchildren. Lepr Rev. 2004;75(1):34-9.

17. Sarmento APA, Pereirão AM, Ribeiro F, Castro JL, Almeida MB, Ramos NM. Perfil epidemiológico da hanseníase no período de 2009 a 2013 no município de Montes Claros (MG), Rev Soc Bras Clin Med. 2015;13(3):180-4.

18. Brito KKG, Araújo DAL, Uchôa REMN, Ferreira JDL, Soares MJGO, Lima JO. Epidemiologia da hanseníase em um estado do Nordeste Brasileiro. Rev Enferm UFPE on line. 2014;8(8):2686-93.

19. Ribeiro Júnior AF, Vieira MA, Caldeira AP. Perfil epidemiológico da hanseníase em uma cidade endêmica no Norte de Minas Gerais, Rev Bras Clin Med. 2012;10(4):272-7.

20. Pereira EVE, Nogueira LT, Machado HAS, Lima LANL, Ramos CHM. Perfil epidemiológico da hanseníase no município de Teresina, no período de 2001 a 2008. An Bras Dermatol. 2011;86(2):235-40.

21. Pereira SVM, Bachion MM, Souza AGC, Vieira SMS. Avaliação da hanseníase: relato de experiência de acadêmicos de enfermagem. Rev Bras Enferm. 2008;61(Esp):774-80.

22. Barcellos C, Bastos FI. Geoprocessamento, ambiente e saúde: uma união possível? Cad Saúde Públ. 1996;12(3):389-97.

\section{Como citar este artigo:}

Monteiro MJSD, Santos GM, Barreto MTS, Silva RVS, Jesus RLR, Silva HJN. Perfil epidemiológico de casos de hanseníase em um estado do nordeste brasileiro. Rev. Aten. Saúde. 2017;15(54):21-28. 